Copyright 2021 Society of Photo-Optical Instrumentation Engineers. One print or electronic copy may be made for personal use only. Systematic reproduction and distribution, duplication of any material in this paper for a fee or for commercial purposes, or modification of the content of the paper are prohibited. 


\title{
Deep Learning for Structural Health Monitoring under Environmental and Operational Variations
}

\author{
Mohsen Mousavi and Amir H. Gandomi ${ }^{\mathrm{a}}$ \\ ${ }^{a}$ Faculty of Engineering and IT, University of Technology Sydney, Ultimo, NSW 2007, \\ Australia
}

\begin{abstract}
Structural Health Monitoring (SHM) techniques can be classified into global and local monitoring strategies. Regarding the global strategies, the aim is to monitor the structure for any change that can be related to damage globally, whereas, the local damage detection schemes usually aim at detecting damage at a very confined area on the structure. The global techniques, which are also sometimes termed as vibration methods, are very sensitive to Environmental and Operational Variations (EOV). These variations can affect the structural response and, subsequently, mask any changes in vibration signals that can be referred to as damage. It is known that temperature has the most effect on the natural frequencies of any structure. In this paper, an inverse strategy is proposed that aims to predict the temperature variation using frequency time series of the structure. To this end, a Recurrent Neural Networks (RNN) is exploited in which the natural frequency time series obtained from the intact structure along with recorded temperatures are respectively used as training features and label. The trined RNN is then tested on data obtained from the damaged structure. It is shown that the error in predictions increases as the damage occurs. A numerical example is presented to demonstrate the applicability of the proposed method.
\end{abstract}

Keywords: Structural Health Monitoring, Deep Learning, Recurrent Neural Network, Long-Short Term Memory Cells, Environmental and Operational Variations

\section{INTRODUCTION}

It is known that the environmental and operational variations affect the structural modal properties and, subsequently, can mask the effect of damage in the structural response, as shown in Ref. 1. This will further make the task of damage detection challenging. This is especially the case when the results of the long-run monitoring of the structure are used for damage detection. For instance, it has been reported that there is a stronger long-term, as opposed to the short-term, correlation between variations of natural frequency and temperatures, as shown in Ref. 2. Therefore, it is important to mitigate the operational and environmental effects in the long-term recorded modal data to avoid false-positive and false-negative damage detection scenarios. Data normalisation, as shown in Ref. 3, and robust regression analysis, as shown in Ref. 4, have been used to address this issue.

Reiterated, it is known that the temperature variations can affect the natural frequencies of a structure. Therefore, having the natural frequencies of the structure and the ambient temperature measured, a deep learning strategy can be developed that can predict the temperature using the natural frequency signals as features. As such, the proposed technique can be considered an inverse strategy where the structural responses are used to predict the temperature signal. To that end, we propose to use Recurrent Neural Networks (RNN) with LongShort Term Memory (LSTM) cells. As such, a portion of the recorded data corresponding to the intact structure is used to train the RNN. The trained RNN is then used to predict the temperature using the natural frequency signals given as features. We show that the error in the predicted temperature signal deviates significantly from its expected value as soon as the damage occurs. Moreover, a signal decomposition technique is used to remove the seasonal patterns in the data to make the task of prediction simpler. To demonstrate the effectiveness of the proposed technique a numerical example is solved and the results are fully discussed in following chapters.

Further author information: (Send correspondence to Amir H. Gandomi)

Amir H. Gandomi: E-mail: gandomi@uts.edu.au

Mohsen Mousavi: E-mail: mohsen.mousavi@uts.edu.au 


\section{PROPOSED METHOD}

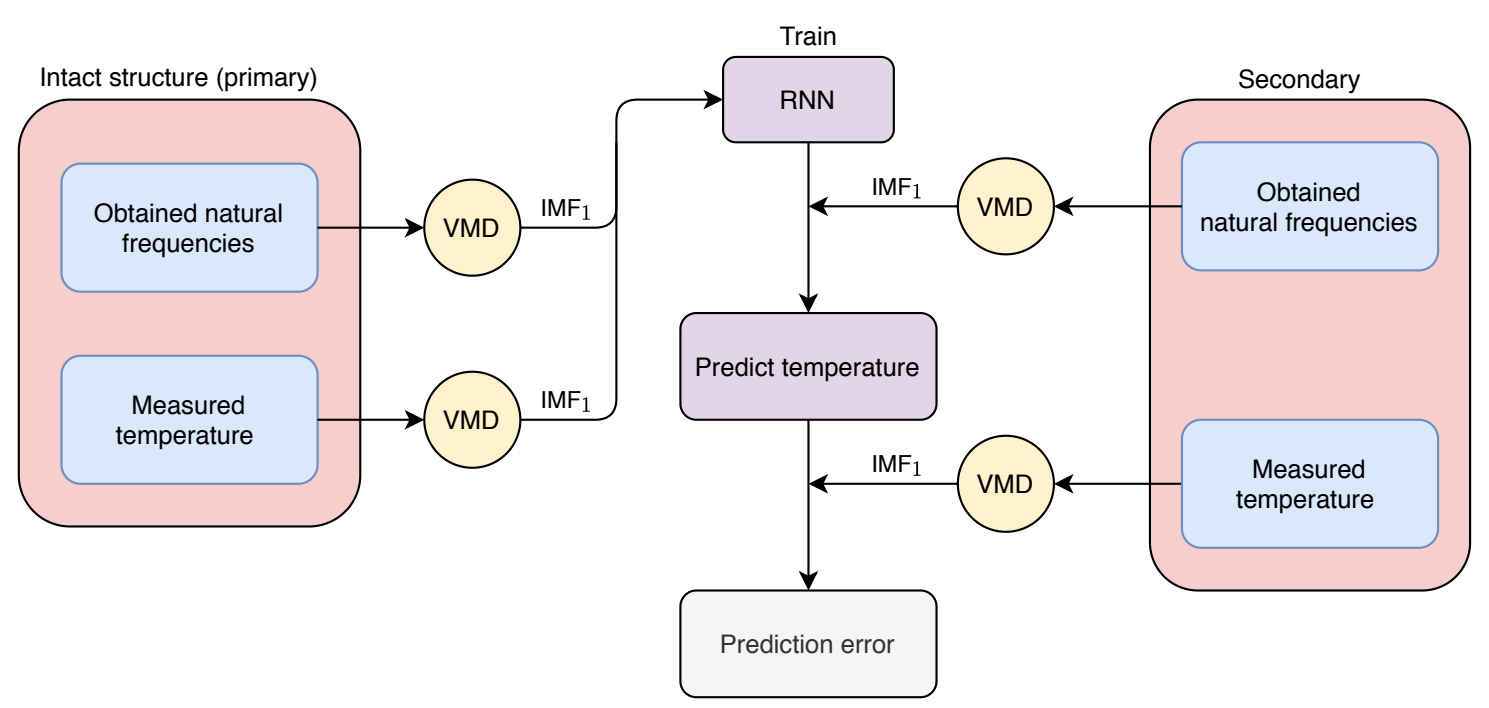

Figure 1: Diagram of the proposed method.

Fig. 1 depicts the procedure of the proposed method. First, the structural vibration data as well as the temperature variation signal corresponding to the intact structure are measured. The next step is to retrieve the structural local modal data (natural frequencies) at the corresponding locations at every time instant. Then, the signals are decomposed using an advanced decomposition technique termed as Variational Mode Decomposition (VMD) into their constituent sub-signals to remove the seasonal variations in all signals. This will further facilitate the procedure of predictions. The obtained natural frequencies and temperature signals (without the seasonal effects) are then used to train a RNN using the former as features and the latter as target.

The trained RNN is then used to predict the value of the temperature using natural frequency signals obtained from the secondary state of the structure. This state can be either a damage or healthy state. In order to determine which state of the structure the secondary collected data corresponds to, the predicted temperature signal without the seasonal effects is compared against its measured counterpart. We hypothesise that the expected value of the prediction errors will deviate from the expected value as damage occurs at the location of the measurements on the structure.

\section{NUMERICAL EXAMPLE AND DISCUSSION}

In this section, the proposed strategy is tested using a numerical example. The numerical example, as such, is a four degree of freedom (DOF) spring-mass system as depicted in Fig. 2.

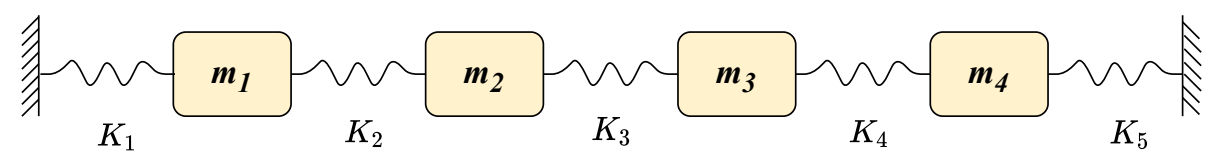

Figure 2: System of mass and springs.

This examples is similar to the one used in Ref. 5 . The masses are $2 \mathrm{~kg}$ each and the stiffness of the springs is obtained as follows,

$$
k_{i}= \begin{cases}-0.11 \times T+4, & \text { if } T<0 \\ -0.03 \times T+4, & \text { if } T \geq 0\end{cases}
$$


for $i=1,2,4,5$, and

$$
k_{3}= \begin{cases}-0.11 \times T+5, & \text { if } T<0 \\ -0.2 \times T+5, & \text { if } T \geq 0\end{cases}
$$

where the unit for stiffness is $k N / m$, and $T$ represents the temperature. Therefore, the stiffness of the springs changes by the temperature. Moreover, the different behaviour of $k_{3}$ introduces non-linearity to the vibration modes.

A temperature profile of one year (from June 2019 to July 2020) hourly recorded temperatures of BaselSwitzerland (Ref. 6) is used in this paper to obtain the stiffness profile of the springs as shown in Fig. 3. Note that the temperature profile used in here is a $-15{ }^{\circ} \mathrm{C}$ shifted real temperature profile to obtain a wider range of negative and positive temperatures. As such, the number of 10000 temperature records are available for which the first four natural frequencies of the spring-mass system are obtained in the same period of time. In order to

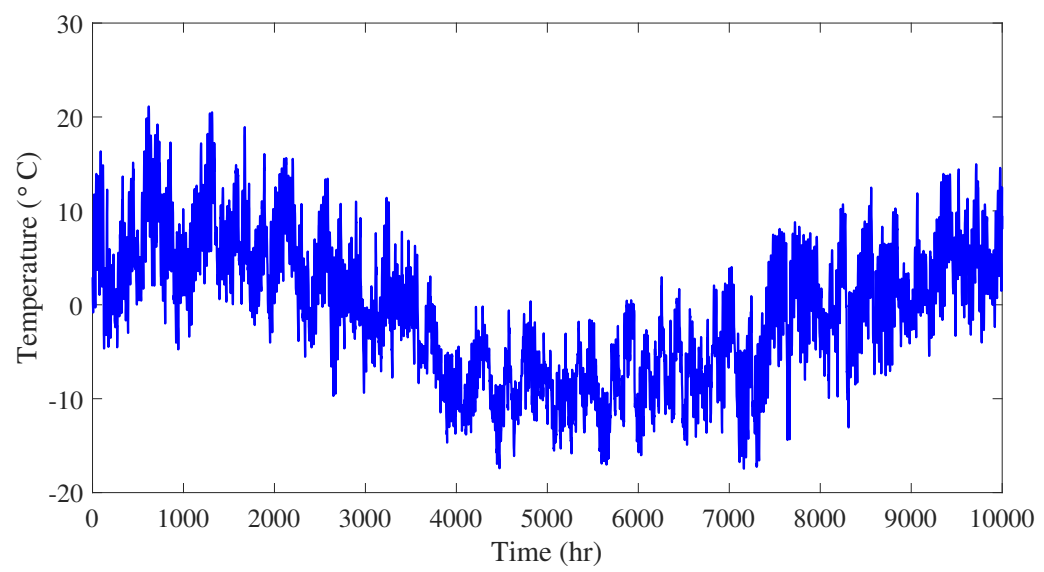

Figure 3: Temperature signal.

simulate damage, it is assumed that the stiffness of $k_{3}$ reduces by $20 \%$ at the $7000^{\text {th }}$ record and this reduction of the stiffness lasts for the duration of 100 records. Therefore, the damage no longer exists after this period which means the damage has been identified and fixed. This has been, in particular, done to ensure that the trained network can be still used for monitoring the structure after the damage is identified and fixed.

In order to simulate the noisy measurements, the obtained natural frequencies of the spring-mass system are contaminated by $10 \%$ noise using the following equation, as shown in Ref. 7 ,

$$
\hat{\delta}=\delta+\frac{\kappa}{100} n_{\text {noise }} \sigma(\delta),
$$

in which, $\delta$ and $\hat{\delta}$ represent noise-free and noisy frequency time series, respectively. $\sigma(\delta)$ and $\kappa$ represent respectively the standard deviation of $\delta$ and the noise level $(=10), n_{\text {noise }}$ is a random independent variables vector of the same length as $\delta$ with a standard normal distribution.

Fig. 4 shows the obtained time series of the four noisy natural frequencies. As evident from the figure, it is very hard, if not impossible, to tell when the damage occurs in the system.

\subsection{Using VMD to remove seasonal effects}

As can be seen from the sub-plots of Fig. 4, there exist some fluctuations in the frequency signals stemming from the seasonal variations of the temperature. These fluctuations do not carry any information about damage. They also make the procedure of the prediction more complicated. Therefore, it is recommended to remove any seasonal effects due to the temperature variations from the frequency signals prior to conducting any further analysis, as shown in Ref. 8. 


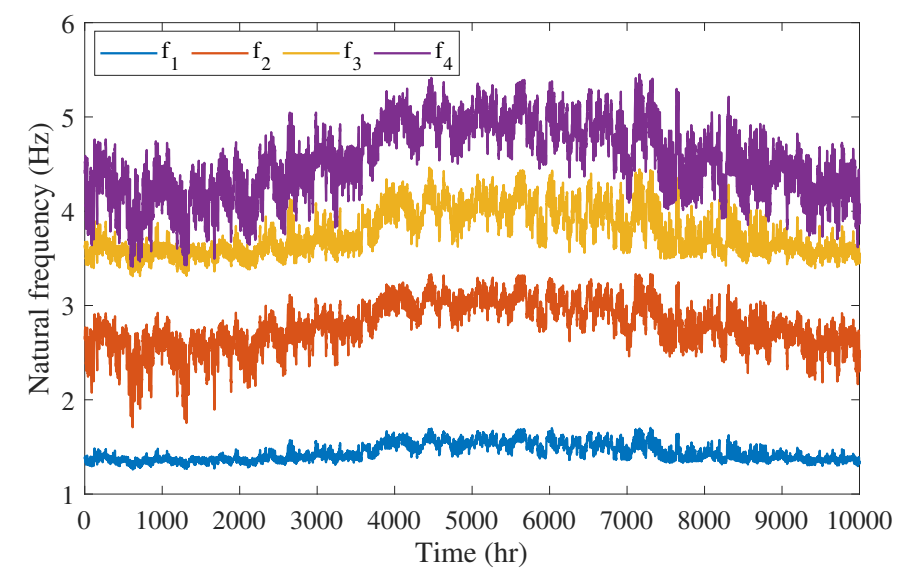

Figure 4: Natural frequencies of the mass-spring system.

In this section, the Variational Mode Decomposition (VMD) algorithm is used to denoise the frequency time series and remove the seasonal patterns in the signals, as shown in Ref. 9. VMD is used to decompose a non-linear non-stationary signal into its constructive modes usually termed as Intrinsic Mode Functions (IMF). Therefore, VMD can preserve any non-linear effects, including those due to the damage, in the frequency signals after decomposition. The obtained IMFs are narrow-band and have a center frequency $\omega$ presented in cycles per unit time.

VMD is a parametric decomposition algorithm and, therefore, there are some parameters that need to be specified prior to the decomposition. Two of the most important of which are the quadratic penalty term and the number of decomposition. The former, which controls how much noise is admitted into the decomposition, is set to 100 in this paper. The number of decomposition is set to two in order to remove the seasonal effect from the long-term non-stationary effect. Another parameter is $D C$ which determines whether or not an IMF is kept at DC level, i.e. a mode with zero center frequency. $D C$ is a Boolean parameter and can take a value of 0 or 1 . In order to ensure that the long-term non-stationary effect of the temperature variations is preserved we choose $D C=1$.

There are, however, other parameters that need to be determined prior to the decomposition. These are: the time step $\tau=0$ which makes the procedure of the denoising effective* ${ }^{*}=10^{-7}$ which is the convergence criteria, and $i n i t=0$ which specifies zero initial values for the center frequencies. Note that init can take other values of 1 or 2 to specify respectively the uniform and random distribution to all the center frequencies. The way of initialising the center frequencies, however, has little effect on the final results, as shown in Ref. 10.

Fig. 5 shows the results of the decomposition. It can be seen that the first IMF, which is of lower center frequency, is kept at DC by setting the parameter $D C=1$ and, therefore, its center frequency is obtained equal to 0 . The results show that average value of the center frequency regarding the second IMFs $\left(\bar{\omega}_{2}\right)$ is obtained about 0.044 cycles per hour. This is equivalent to almost a full cycle per 24 hours, confirming that this mode corresponds to the seasonal effect (temperature variation between day and night) and do not carry any information about the damage. Therefore, they can be excluded from the signals. Likewise, the temperature signal is also decomposed into two modes, setting the decomposition parameters as above. Fig. 6 shows the decomposition results. As can be seen from the figure the center frequency of the $\mathrm{IMF}_{1}$ is obtained equal to 0 as expected. The center frequency of the $\mathrm{IMF}_{2}$ is obtained close to $\bar{\omega}_{2}$, i.e. about 0.043 . Therefore, the first modes of the frequency and temperature signals are used for training a RNN to be further used to predict the temperature using the obtained frequencies.

${ }^{*}$ Note that one can specify $\tau$ to a small value, say 0.1 , if an exact reconstruction is intended. In such a case, the denoising becomes ineffective. 


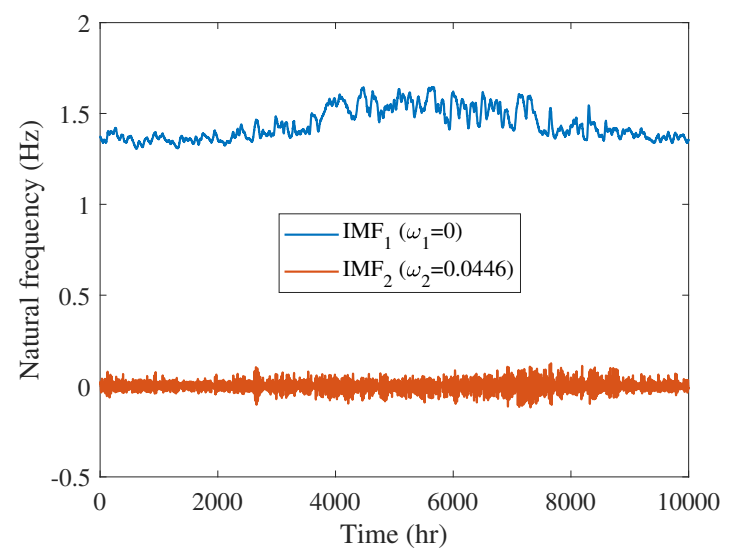

(a) $1^{\text {st }}$ frequency

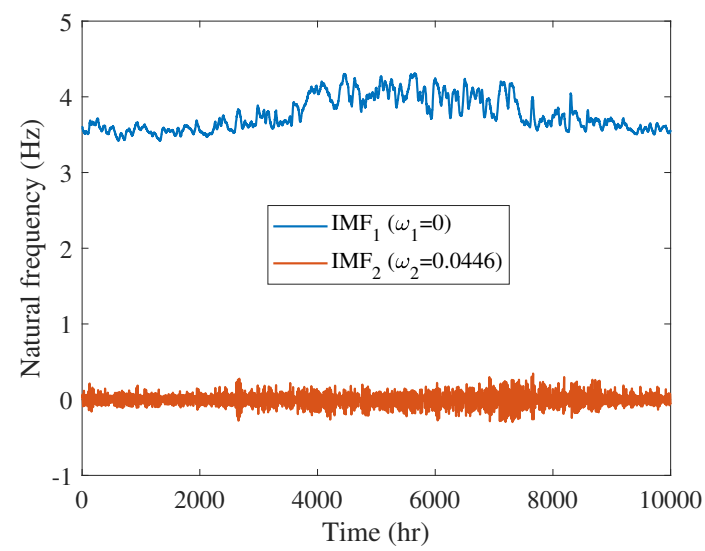

(c) $3^{\text {rd }}$ frequency

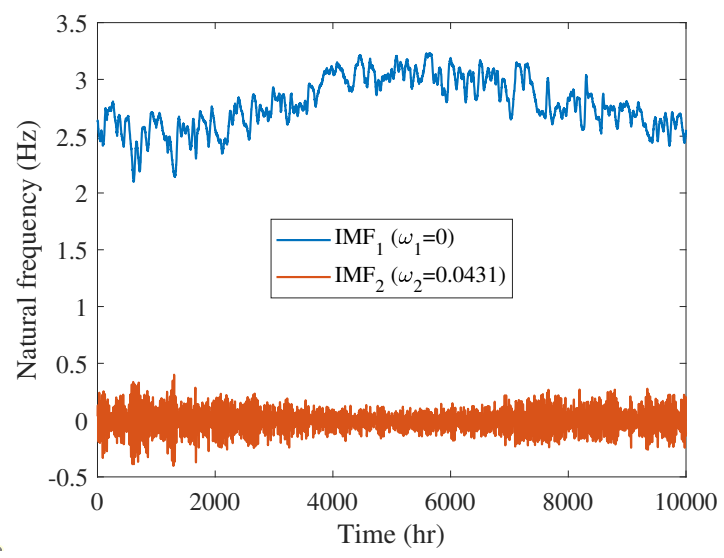

(b) $2^{\text {nd }}$ frequency

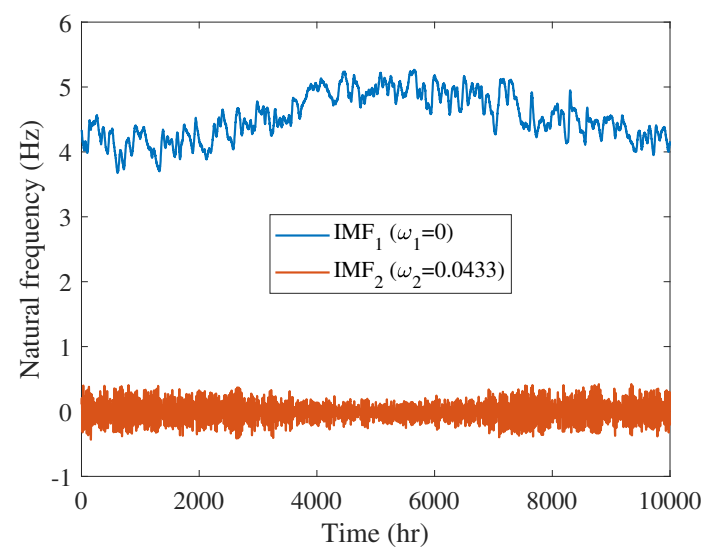

(d) $4^{\text {th }}$ frequency

Figure 5: Decomposed natural frequencies of the spring-mass system along with their center frequencies. The first IMF in all cases is kept at DC (zero center frequency).

\subsection{Using a RNN for training}

In this section, a multivariate stacked Recurrent Neural Network (RNN) is used for training purpose to learn the $\mathrm{IMF}_{1}$ corresponding to the temperature signal using $\mathrm{IMF}_{1}$ obtained from the frequency signals. The architecture of the RNN used in this paper follows,

1. a sequence input layer taking four features as inputs (first IMF of the frequency signals).

2. a LSTM layer with 100 units.

3. a fully connected layer with 50 units.

4. a fully connected layer with one output unit (first IMF of the temperature signal).

The network settings are specified as follows,

1. Adam optimisation is selected as the optimisation algorithm, as shown in Ref. 11.

2. the learning rate is initially set at 0.005 and chosen to decrease by a factor of 0.2 at every 200 epochs. 

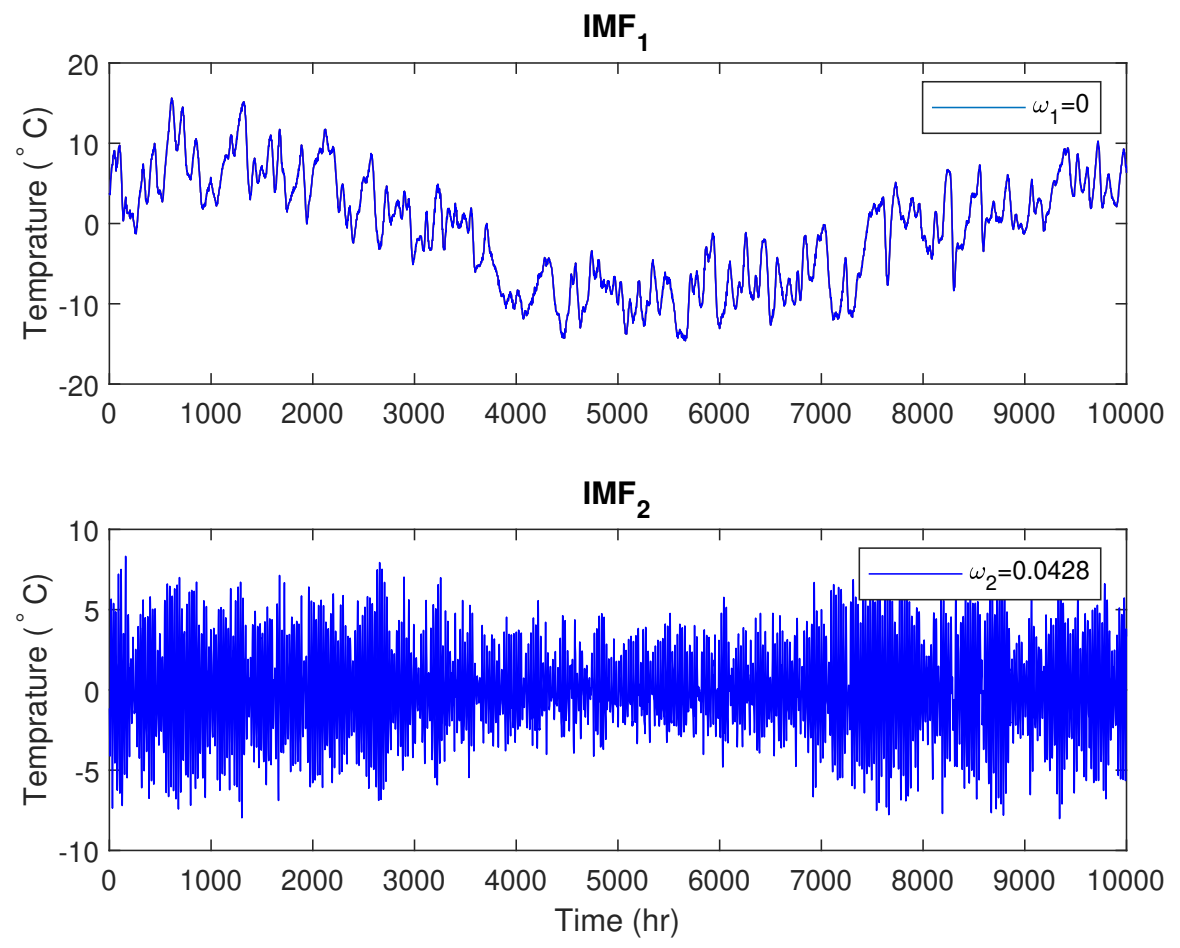

Figure 6: Decomposed temperature signal.

3. the number of maximum epochs is chosen 1000 .

4. a gradient threshold of 1 is considered to avoid exploding gradients effect.

Fig. 7 shows the results of the training and predictions. As can be seen from the figure, $50 \%$ of the available data corresponding to the healthy condition of the spring-mass system is considered as training-set. The remaining dataset is considered as the test-set. A peak is evident in the predicted error at the time when damage occurs in the system. The peak also lasts so long as the damage exists. It is also evident that the error returns to normal as the damage is removed. It can be seen from the figure that the peak is significant, showing that the proposed strategy can detect the damage very effectively and can be used as a method to identify any changes in the frequency signals which can be referred to as damage. This is while one cannot identify the occurrence of the damage through visual inspection of the frequency signals.

\section{CONCLUSIONS AND FUTURE WORK}

A systematic damage identification algorithm has been proposed in this paper that can be used for raising an early alarm as soon as a damage occurs in a structure. The data required for the proposed strategy are a couple of first natural frequency time series of the structure obtained from the vibration signals measured at some time instants as well as the temperature signal measured at the same period of time. It is known that the frequency signals are sensitive to the temperature variations. Accordingly, any changes in these signals due to the damage can be masked by the EOV effects. Therefore, It has been proposed that, using the obtained frequency at each time instant, one can monitor the prediction errors of the temperature signal for damage. It is first recommended, however, that the seasonal effects of all signals to be removed. To that end, the frequency and temperature signals were first decomposed using a nonlinear/non-stationary decomposition algorithm, i.e. VMD. The first IMFs of the decomposition process are then used to train a stacked multivariate RNN. The RNN is trained on the $\mathrm{IMF}_{1} \mathrm{~s}$ corresponding to the frequency signals to learn how to predict the $\mathrm{IMF}_{1}$ of the 

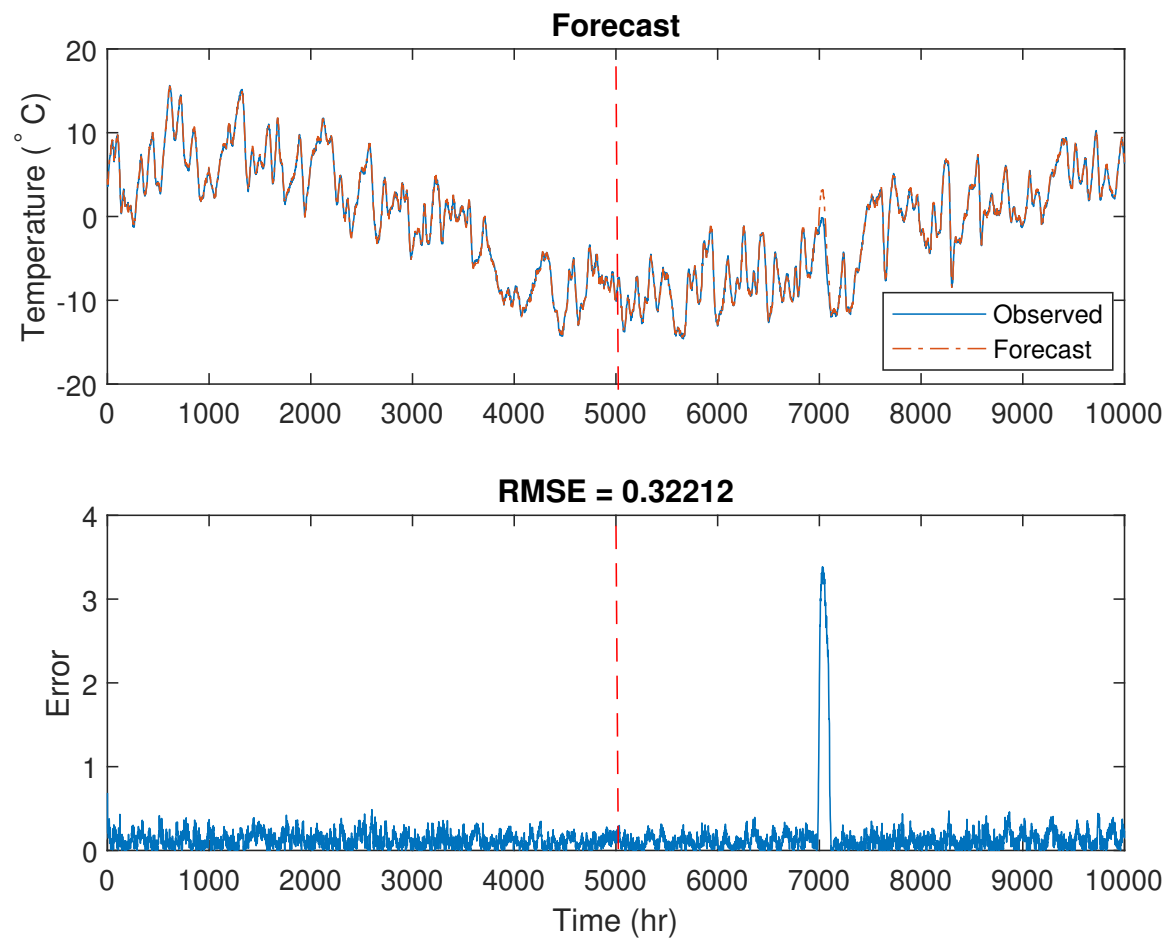

Figure 7: The predicted and observed $\mathrm{IMF}_{1}$ corresponding to the temperature signal along with the prediction errors. The vertical dashed line determines the border between the training and test dataset.

temperature signal. A portion of the dataset corresponding to the healthy state of the structure have been used as the training-set, and the remaining were used as the test-set. The results show that the temperature prediction error deviates significantly from the expected value of the error as the damage occurs. The obtained peak at the time of the occurrence of the damage is also significant. This means that an early alarm can be raised using the proposed strategy as soon as damage occurs in the system.

Moreover, a nonlinear effect was introduced to the studied mass-spring system stemming from different behaviour of spring $k_{3}$. The results show that the proposed method is fairly robust to the existence of such a nonlinear behaviour. As such, the damage detection procedure was still fairly successful. This further demonstrates the applicability of the proposed method to some real-world problems in which a nonlinear correlation between the temperature variations and frequency signals exists.

The proposed method has been tested on a numerical model, i.e a spring-mass system. Therefore, future work need to be conducted on real structures to confirm the effectiveness of the proposed strategy.

\section{REFERENCES}

[1] Liu, C. and DeWolf, J. T., "Effect of temperature on modal variability of a curved concrete bridge under ambient loads," Journal of structural engineering 133(12), 1742-1751 (2007).

[2] Feltrin, G., "Temperature and damage effects on modal parameters of a reinforced concrete bridge," in [Proc., 4th Int. Conf. on Structural Dynamics, Eurodyn 2002], 373-378, Balkema (2002).

[3] Farrar, C. R., Sohn, H., and Worden, K., "Data normalization: a key for structural health monitoring," tech. rep., Los Alamos National Laboratory (2001).

[4] Dervilis, N., Worden, K., and Cross, E., "On robust regression analysis as a means of exploring environmental and operational conditions for shm data," Journal of Sound and Vibration 347, 279-296 (2015). 
[5] Shi, H., Worden, K., and Cross, E. J., "A regime-switching cointegration approach for removing environmental and operational variations in structural health monitoring," Mechanical Systems and Signal Processing 103, 381-397 (2018).

[6] "Weather history download basel," (Retrieved August 18, 2020).

[7] He, W.-Y., Ren, W.-X., and Zhu, S., "Damage detection of beam structures using quasi-static moving load induced displacement response," Engineering Structures 145, 70-82 (2017).

[8] Shi, H., Worden, K., and Cross, E. J., "A cointegration approach for heteroscedastic data based on a time series decomposition: an application to structural health monitoring," Mechanical Systems and Signal Processing 120, 16-31 (2019).

[9] Dragomiretskiy, K. and Zosso, D., "Variational mode decomposition," IEEE Transactions on Signal Processing 62(3), 531-544 (2014).

[10] Wang, Y., Markert, R., Xiang, J., and Zheng, W., "Research on variational mode decomposition and its application in detecting rub-impact fault of the rotor system," Mechanical Systems and Signal Processing $\mathbf{6 0}$, 243-251 (2015).

[11] Kingma, D. P. and Ba, J., "Adam: A method for stochastic optimization," arXiv preprint arXiv:1412.6980 (2014). 